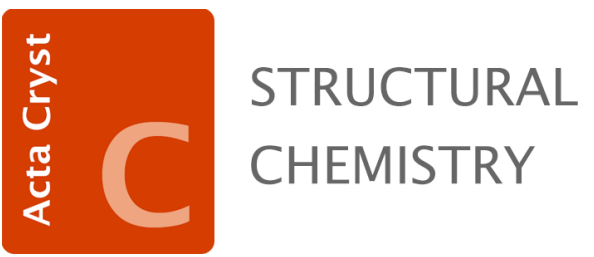

Volume 72 (2016)

Supporting information for article:

(H3O)3Sb2Br9: the first member of the M3E2X9 structure family with oxonium cations

Jen-Hui Chang, Thomas Doert and Michael Ruck 


\section{S1. Degree of distortion}

The degree of distortion $(d d)$ was defined from the dispersion of $d\left(M^{\cdots} \cdots M^{\prime}\right)(E q . \mathrm{S} 1-\mathrm{S} 3) . d\left(M^{\cdots} \cdots M^{\prime}\right)$ are the distances of the $M$ atom centering the hexagonal star to the six neighboring $M^{\prime}$ atoms. (Chang, et al. 2016).

$$
\begin{aligned}
& \bar{d}=\sum d\left(M^{\cdots} M^{\prime}\right) / 6 \\
& \Delta d=\left[d\left(M \cdots M^{\prime}\right)_{\max }-d\left(M^{\prime} \cdots M^{\prime}\right)_{\min }\right] \\
& d d(\text { degree of distortion }) \equiv \Delta d / 2 \bar{d}
\end{aligned}
$$

Factor 2 in $E q$. (S3) is used for normalization. 


\section{Figure S1}

EDX spectrum of the single crystal recorded after the XRD experiment. The crystal was mounted on a carbon pad of an Al sample holder. Signals of Cs atoms were not detected (expected energies of Cs signals are marked in red).

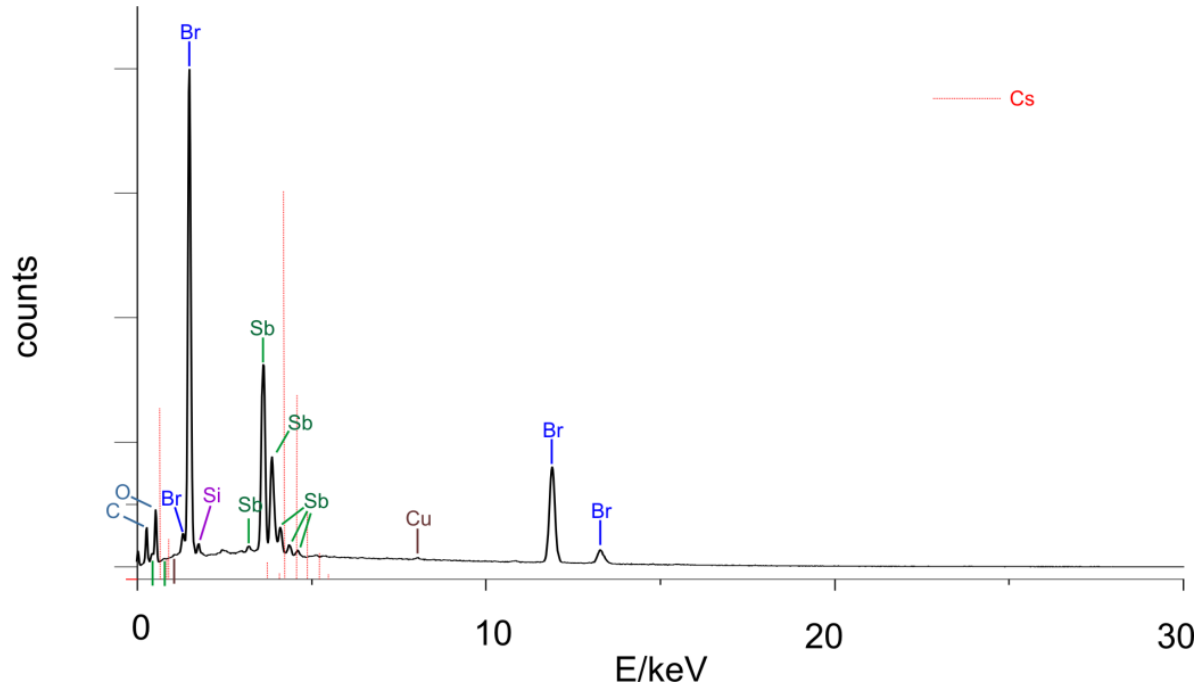

